\title{
Preventive Effect of Clazosentan against Cerebral Vasospasm after Clipping Surgery for Aneurysmal Subarachnoid Hemorrhage in Japanese and Korean Patients
}

\author{
Miki Fujimura $^{a}$ Jin-Yang Joo ${ }^{c}$ Jong-Soo Kim ${ }^{d}$ Motonori Hattab \\ Yoshinari Yokoyama ${ }^{b}$ Teiji Tominaga ${ }^{a}$ \\ a Department of Neurosurgery, Tohoku University Graduate School of Medicine, Sendai, and \\ ${ }^{b}$ Actelion Pharmaceuticals Japan Ltd., Tokyo, Japan; ' Department of Neurosurgery, Cerebrovascular Center, \\ Gangnam Severance Hospital, Yonsei University College of Medicine, and d Department of Neurosurgery, \\ Samsung Medical Center, Seoul, South Korea
}

\section{Keywords}

Clazosentan · Clipping · Delayed ischemic neurological deficit - Subarachnoid hemorrhage · Endothelin antagonist · Randomized trial · Vasospasm

\begin{abstract}
Background: Clazosentan has been explored worldwide for the prophylaxis of cerebral vasospasm after aneurysmal subarachnoid hemorrhage (aSAH). In a dose-finding trial (CONSCIOUS-1) conducted in Israel, Europe, and North America, clazosentan (1, 5, and $15 \mathrm{mg} / \mathrm{h}$ ) significantly reduced the incidence of cerebral vasospasm, but its efficacy in Japanese and Korean patients was unknown. We conducted a double-blind comparative study to evaluate the occurrence of cerebral vasospasm in Japanese and Korean patients with aSAH. Methods: The aim of this multicenter, double-blind, randomized, placebo-controlled, dose-finding phase 2 clinical trial, was to evaluate the efficacy, pharmacokinetics, and safety of clazosentan (5 and $10 \mathrm{mg} / \mathrm{h}$ ) against cerebral vasospasm after clipping surgery in Japanese and Korean patients with aSAH. Patients aged between 20 and 75 years were administered the study drug within $56 \mathrm{~h}$ after the aneurysm rupture and up to day 14 post-aSAH. The inci-
\end{abstract}

\section{KARGER}

E-Mail karger@karger.com www.karger.com/ced
(C) 2017 The Author(s)

Published by S. Karger AG, Basel

This article is licensed under the Creative Commons AttributionNonCommercial-NoDerivatives 4.0 International License (CC BYNC-ND) (http://www.karger.com/Services/OpenAccessLicense). Usage and distribution for commercial purposes as well as any distribution of modified material requires written permission. dence of vasospasm, defined as an inner artery diameter reduction of major intracranial arteries $\geq 34 \%$ based on catheter angiography, was compared between each treatment group. Cerebral infarction due to vasospasm at 6 weeks and patients' outcome at 3 months was also compared. Results: Among 181 enrolled patients, 158 completed the study and were analyzed. The incidence of vasospasm up to day 14 after aSAH onset was $80.0 \%$ in the placebo group $(95 \% \mathrm{Cl} 67.0$ 89.6 ), $38.5 \%$ in the $5 \mathrm{mg} / \mathrm{h}$ clazosentan group (95\% Cl $25.3-$ $53.0)$, and $35.3 \%$ in the $10 \mathrm{mg} / \mathrm{h}$ clazosentan group $(95 \% \mathrm{Cl}$ 22.4-49.9), indicating that the incidence of vasospasm was significantly reduced by clazosentan treatment (placebo vs. $5 \mathrm{mg} /$ h clazosentan, $p<0.0001$; placebo vs. $10 \mathrm{mg} / \mathrm{h}$ clazosen$\tan , p<0.0001)$. The occurrence of cerebral infarction due to vasospasm was $20.8 \%$ in the placebo group (95\% Cl $10.8-$ $34.1), 3.8 \%$ in the $5 \mathrm{mg} / \mathrm{h}$ clazosentan group $(95 \% \mathrm{Cl} 0.5-$ $13.2)$, and $4.2 \%$ in the $10 \mathrm{mg} / \mathrm{h}$ clazosentan group $(95 \% \mathrm{Cl}$ 0.5-14.3), indicating that clazosentan significantly reduced the occurrence of cerebral infarctions caused by vasospasm (placebo vs. $5 \mathrm{mg} / \mathrm{h}$ clazosentan, $p=0.0151$; placebo vs. 10 $\mathrm{mg} / \mathrm{h}$ clazosentan, $p=0.0165)$. The overall incidence of allcause death and/or vasospasm-related morbidity/mortality was significantly reduced in the $10 \mathrm{mg} / \mathrm{h}$ clazosentan group compared with the placebo group ( $p=0.0003)$. Conclusion:

Miki Fujimura, MD, $\mathrm{PhD}$

Department of Neurosurgery

Tohoku University Graduate School of Medicine

1-1 Seiryo-machi, Aoba-ku, Miyagi, Sendai 980-8574 (Japan)

E-Mail fujimur@nsg.med.tohoku.ac.jp 
These results suggest that clazosentan prevents cerebral vasospasm and subsequent cerebral infarction, and could thereby improve outcomes after performing a clipping surgery for aSAH in Japanese and Korean patients.

(c) 2017 The Author(s)

Published by S. Karger AG, Basel

\section{Introduction}

Prevention of cerebral vasospasm is one of the most critical factors to improve the outcome of clipping surgery in patients with aneurysmal subarachnoid hemorrhage (aSAH). Once cerebral vasospasm develops, patients experience delayed ischemic neurological deficit (DIND) in $17-40 \%$ of cases, half of which result in cerebral infarction [1]. Nimodipine has been used worldwide for improvement in neurological outcomes, and a variety of drugs such as fasudil hydrochloride (Rho kinase inhibitor) and sodium ozagrel (thromboxane A2 synthase inhibitor) have been used for the prevention of vasospasm [2, 3]. However, despite the use of these drugs, it is still difficult to prevent cerebral vasospasm efficiently. It is therefore necessary to develop an alternative prophylactic agent against cerebral vasospasm [2].

In aSAH, the release of oxyhemoglobin-derived endothelin from red blood cells is believed to contribute significantly to the development of cerebral vasospasm due to its potent, long-lasting vasoconstrictor effects [4-6]. In light of the potential role of endothelin in vasospasm, clazosentan an endothelin-A receptor antagonist - has been investigated worldwide as a prophylactic agent to prevent cerebral vasospasm [7-9]. The "Clazosentan to overcome neurological ischemia and infarction occurring after subarachnoid hemorrhage" (CONSCIOUS)-1 trial demonstrated that the incidence of cerebral vasospasm was significantly reduced by clazosentan in Israeli, European, and North American patients [7]. However, theefficacy of clazosentan in Japanese and Korean patients was unknown, since Japan and Korea were not included. Therefore, we conducted a phase- 2 dose-finding trial to evaluate the use of clazosentan in Japanese and Korean patients. Clazosentan was administered at doses of 5 and $10 \mathrm{mg} / \mathrm{h}$, which are different from the doses used in the CONSCIOUS- 1 trial to account for the smaller body sizes of Asian patients compared with European and American patients [10].

\section{Methods}

\section{Study Design}

The study was a multicenter, double-blind, randomized, placebo-controlled, dose-finding, phase- 2 trial to investigate the occurrence of cerebral vasospasm in patients with aSAH who had undergone clipping surgery. Patients were administered either 5 or $10 \mathrm{mg} / \mathrm{h}$ clazosentan or a matching placebo (Fig. 1). The study was conducted by specialized neurosurgeons in 22 centers in Japan and 10 centers in South Korea.

The study was approved by the Institutional Review Boards of each center and was conducted in accordance with the Declaration of Helsinki and Good Clinical Practice guidelines (Trial Registration: Japan Pharmaceutical Information Center Clinical Trial Information [JapicCTI-152889]). Prior to the start of the trial, physicians fully explained the methods and objectives of the trial to patients and/or their legal guardians, and obtained voluntary written consent for each patient.

\section{Population}

Participating patients were Japanese or Korean individuals between the ages of 20 and 75 years. The main inclusion criteria were classification as group 3 on the Fisher scale and long axis of subarachnoid hemorrhage of $\geq 20 \mathrm{~mm}$ or extending to both hemispheres of the brain on the preoperative CT scan. The main exclusion criteria were as follows: being classified as World Federation of Neurosurgical Surgeons (WFNS) grade 5; serious intraoperative complications such as large territorial cerebral infarction observed; hemiplegia or disturbance of consciousness continuing for $\geq 12 \mathrm{~h}$ after clipping surgery; experiencing treatment-resistant hy-
Fig. 1. Study design. aSAH, aneurysmal subarachnoid hemorrhage; DSA, digital subtraction angiography; GOSE, Glasgow Outcome Scale-Extension; mRS, modified Rankin Scale; MMSE, Mini-Mental State Examination.

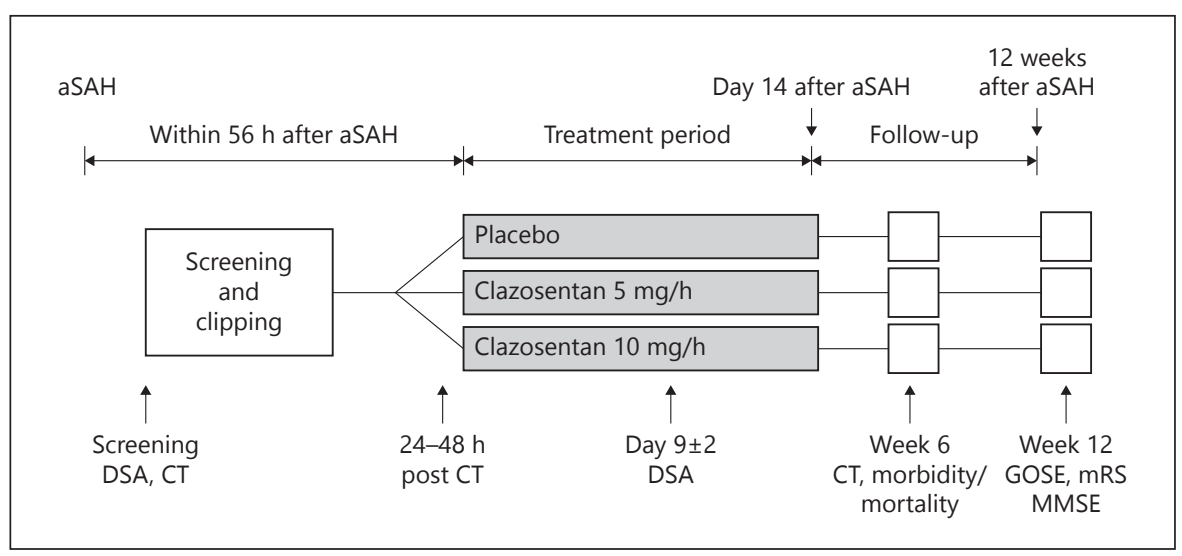

60

Cerebrovasc Dis 2017;44:59-67 DOI: $10.1159 / 000475824$
Fujimura/Joo/Kim/Hatta/Yokoyama/ Tominaga 
potension; severe heart failure; aspiration pneumonia or pulmonary edema; a serum creatinine level of $\geq 2.0 \mathrm{mg} / \mathrm{dL}$; a total bilirubin level of more than twice the upper limit of the facility's reference value; and evidence of liver cirrhosis. We did not include patients in groups 1,2, and 4 on the Fisher group.

\section{Study Intervention}

Clazosentan or placebo administration was started within $56 \mathrm{~h}$ after the onset of aSAH and continued up to day 14 after the onset of aSAH.

Concomitant use of fasudil hydrochloride (not approved for use in South Korea), sodium ozagrel, nizofenone fumarate (release canceled in South Korea), ticlopidine hydrochloride (not approved for use in South Korea), intravenous (i.v.) nimodipine (not approved for use in Japan), i.v. nicardipine, or statins (acceptable if administration was started before onset of aSAH) was prohibited. However, no restriction was placed on the use of oral nimodipine, which is approved for use in South Korea.

\section{Clinical Assessment and Radiology}

Digital subtraction angiography (DSA) and CT imaging were conducted for all patients (Fig. 1). After anonymization, images were submitted for evaluation by an Image Review Committee (IRC) comprising 5 radiology or neurosurgical specialists. The IRC members measured the inner artery diameters of the proximal blood vessels, and assessed the qualitative severity of cerebral vasospasm for distal blood vessels based on an inner artery reduction $<34 \%$ (none to mild), 34-65\% (moderate), and $\geq 66 \%$ (severe; Fig. 2). Based on previous observations, the presence and severity of vasospasm according to these categories correlated with the development of brain ischemia and cerebral infarction $[11,12]$. Any 2 IRC members assessed the same case independently and if there was a discrepancy in their findings, the committee chairperson gave a final judgment. Trans-cranial Doppler ultrasound was not used for the assessment of vasospasm in this study.

\section{Efficacy}

The primary endpoint was a comparison of the occurrence of moderate or severe cerebral vasospasm up to day 14 after aSAH onset between the placebo and clazosentan groups. The secondary endpoints were the occurrence of newly formed cerebral infarctions associated with vasospasm within 6 weeks after aSAH onset, and assessments of Glasgow Outcome Scale-Extended (GOSE), modified Rankin Scale (mRS), and Mini-Mental State Examination (MMSE) at 12 weeks after aSAH onset.

The definition of a poor outcome included a GOSE score of $\leq 4$ and an $\mathrm{mRS}$ score of $\geq 3$. The exploratory endpoint was an assessment of the occurrence of morbidity/mortality (M/M) events within 6 weeks after aSAH, which was a composite endpoint. An $\mathrm{M} / \mathrm{M}$ event was defined as death for any reason, a vasospasm-related cerebral infarction, a vasospasm-related DIND, or the start of rescue therapy for vasospasm.

A DIND was recorded based on different criteria depending on the availability of neurological assessment findings. For patients capable of undergoing neurological assessment, a DIND was confirmed based on a decrease of $\geq 2$ points on the modified Glasgow Coma Scale or an increase of $\geq 2$ points on the abbreviated National Institutes of Health Stroke Scale lasting for $\geq 2$ h. For patients whose neurological scores could not be assessed, a DIND was recorded based on clinical findings suggest- ing cerebral vasospasm (e.g., somnolence and fever) or, in the absence of such findings when cerebral vasospasm was observed on a DSA examination and rescue therapy was initiated.

Rescue therapy involved HHH therapy (hypervolemia, hypertension, and hemodilution), percutaneous transluminal angioplasty, and intra-arterial administration of anti-platelet drugs (e.g., ozagrel) or vasodilators (e.g., fasudil hydrochloride, papaverine hydrochloride, and nimodipine) in the presence of moderate or severe vasospasm on DSA or clinical symptoms suggestive of vasospasm.

\section{Pharmacokinetics}

The aims of pharmacokinetics measurements were: (1) to compare the plasma clazosentan concentration between the $5 \mathrm{mg} / \mathrm{h}$ and the $10 \mathrm{mg} / \mathrm{h}$ dose groups and (2) to compare differences in clazosentan pharmacokinetics between Japanese and Korean patients. Blood samples were collected at a steady state on day $9 \pm 2$ after aSAH. The pharmacokinetics of clazosentan has previously been reported to be similar in Japanese and Caucasian patients [13].

\section{Safety}

All adverse events that occurred between the start of the study drug administration and $24 \mathrm{~h}$ after discontinuation or completion of study treatment were recorded. Notable adverse events including pulmonary complications, hypotension, and anemia were recorded from the start of study drug administration up to 6 weeks after aSAH onset. In addition, mortalities that occurred up to 12 weeks after aSAH onset were recorded.

\section{Statistical Analysis}

Sample size was calculated based on the phase 2 a clinical trial with clazosentan [14] and the occurrence of cerebral vasospasm in the CONSCIOUS- 1 phase 2 dose-finding trial [7]. It was set to the number of patients necessary to obtain a power of 0.9 at a 2 -sided significance level of $5 \%$.

The per-protocol set (PPS) was used for primary efficacy analysis in order to most reliably detect any dose-response relationship for the target population from whom valid data were available. Analysis was also conducted based on the all-treated set (ATS), which comprised patients who had received the study drug at least once and had undergone at least one efficacy evaluation after drug administration. The safety analysis group (safety set) included all patients who received at least one dose of study drug and underwent at least one safety evaluation thereafter.

Patients were assigned to groups by stratified randomization. The stratification factors were country (Japan vs. South Korea) and WFNS grade at the time of screening (I and II, vs. III and IV). The group assignment schedule was managed by a registration center and was not made known to anyone involved in the trial (except those involved in the work at the registration center) until unblinding was done.

Fisher's exact test was used to assess the occurrence of cerebral vasospasm, and the Bonferroni-Holm method was used to adjust multiple testing. Patients with missing data were excluded from the analysis. In a sensitivity analysis to examine the effect of missing values, patients in whom the presence of cerebral vasospasm could not be determined by the IRC were assigned imputed findings of "severe cerebral vasospasm." 


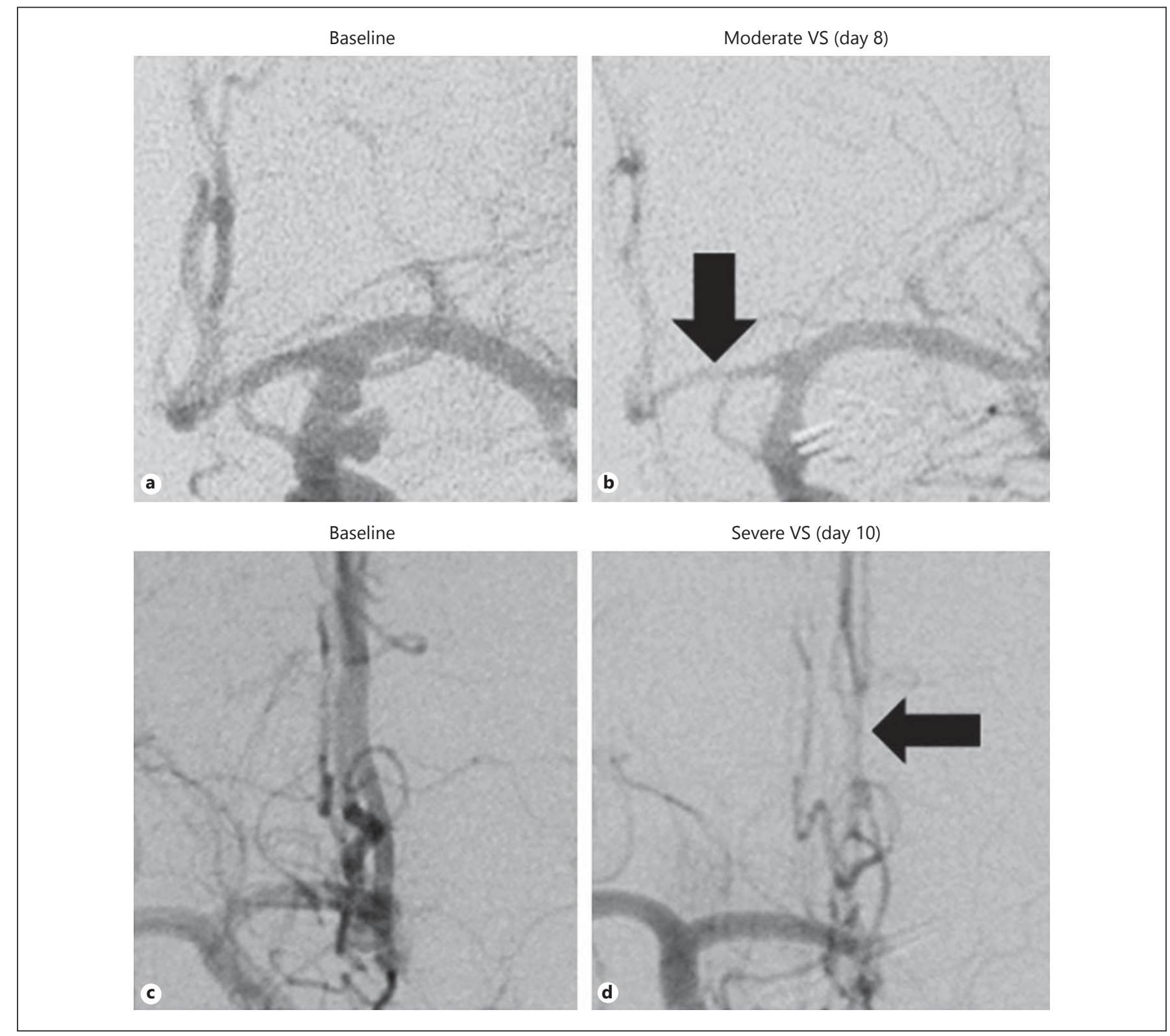

Fig. 2. Digital subtraction angiography images taken before and after the occurrence of moderate, severe cerebral vasospasm (VS). Patient 1 (aged 55 years): image taken (a) before clipping surgery and (b) 8 days after aneurysmal subarachnoid hemorrhage (aSAH)

The occurrence of cerebral infarction associated with vasospasm within 6 weeks after aSAH onset, as well as GOSE and mRS scores, were assessed for a dose-response relationship using the Cochran-Armitage trend test. A Jonckheere-Terpstra test was conducted to assess the results of the MMSEs.

Analysis of variance was used to assess differences in clazosentan pharmacokinetics between countries and dose groups.

SAS ${ }^{\circledR}$ software version 9.2 (SAS Institute, Cary, NC, USA) was used for conducting statistical analyses. onset; spasm rate $34-65 \%$. Patient 2 (aged 51 years): image taken (c) before clipping surgery and (d) 10 days after aSAH onset; spasm rate: $\geq 66 \%$. Both patients received placebo.

\section{Results}

\section{Patients}

Patients were recruited for the trial from June 2009 to November 2010. Initially, 181 patients were enrolled. However, 2 patients were not administered the study drug because 1 patient withdrew consent and the other violated the inclusion/exclusion criteria. Therefore, the 


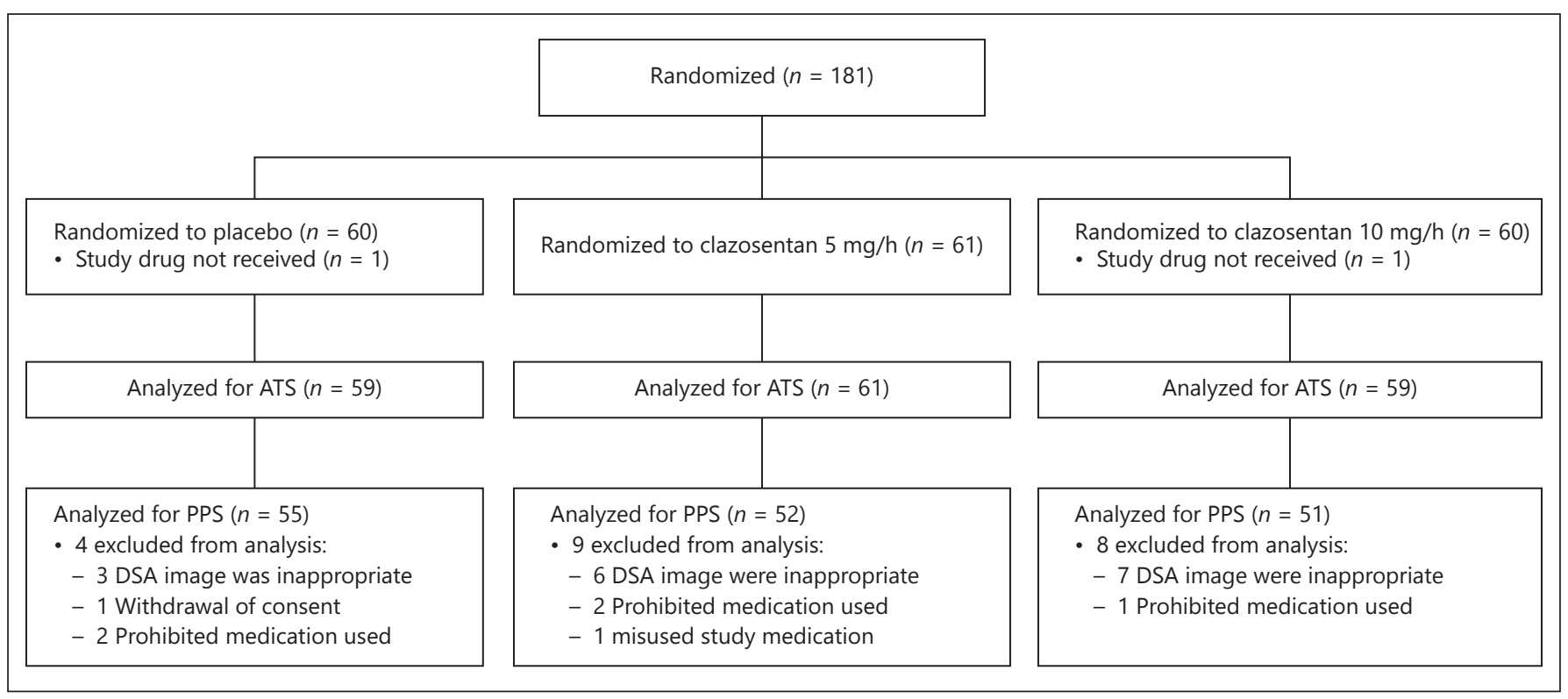

Fig. 3. Patient disposition. Two placebo patients had more than one reason for exclusion from the PPS. ATS, all-treated set; DSA, digital subtraction angiography; PPS, per-protocol set.

ATS comprised 179 individuals (Fig. 3). Of the patients who reached the drug administration stage of the study, one who was randomized to $5 \mathrm{mg} / \mathrm{h}$ clazosentan was administered placebo during the entire treatment period in error. This patient was assessed as part of the $5 \mathrm{mg} / \mathrm{h}$ group in the ATS efficacy evaluation. In the safety set $(n=179)$, this patient was assessed as part of the placebo group. The PPS comprised 158 patients (Fig. 3). The most common 2 reasons for patient exclusion from the PPS were inappropriate DSA imaging methodology and concomitant use of prohibited medications.

In the placebo, $5 \mathrm{mg} / \mathrm{h}$ clazosentan, and $10 \mathrm{mg} / \mathrm{h}$ clazosentan groups, study drug administration was discontinued in $13.6 \%(8 / 59), 11.5 \%(7 / 61)$, and $6.8 \%(4 / 59)$ patients, respectively, mainly due to adverse events (13/19) or concomitant use of prohibited drugs (3/19).

No significant differences in patient demographics were observed among the groups studied (Table 1).

\section{Efficacy}

Moderate or severe cerebral vasospasm up to day 14 after the onset of aSAH occurred in $80.0 \%$ of patients inthe placebo group (95\% CI 67.0-89.6), 38.5\% in the $5 \mathrm{mg} / \mathrm{h}$ clazosentan group (95\% CI 25.3-53.0), and 35.3\% in the $10 \mathrm{mg} / \mathrm{h}$ clazosentan group (95\% CI 22.449.9; Fig. 4). Moderate or severe vasospasm was significantly reduced with both doses of clazosentan (placebo vs. $5 \mathrm{mg} / \mathrm{h}$ clazosentan, $p<0.0001$; placebo vs. $10 \mathrm{mg} / \mathrm{h}$

Clazosentan Prevents Cerebral Vasospasm after Clipping Surgery for aSAH clazosentan, $p<0.0001$; significance level 0.025 using the Bonferroni-Holm method). Findings from the ATS were supportive of the primary PPS analysis.

The incidence of cerebral infarction associated with vasospasm up to 6 weeks after aSAH onset was $20.8 \%$ in the placebo group (95\% CI 10.8-34.1), 3.8\% in the $5 \mathrm{mg} / \mathrm{h}$ clazosentan group (95\% CI $0.5-13.2)$, and $4.2 \%$ in the $10 \mathrm{mg} / \mathrm{h}$ clazosentan group (95\% CI 0.5-14.3; Fig. 5). Compared with placebo, the incidence of cerebral infarction associated with vasospasm was significantly lower in both clazosentan groups (placebo vs. $5 \mathrm{mg} / \mathrm{h}$ clazosentan, $p=0.0151$; placebo vs. $10 \mathrm{mg} / \mathrm{h}$ clazosentan, $p=0.0165$ ). There were no significant differences in GOSE and mRS scores at 12 weeks after aSAH onset between either clazosentan dose group and the placebo group (Fig. 5). The mean MMSE scores were identical across all 3 treatment groups.

The occurrence of $\mathrm{M} / \mathrm{M}$ events was $47.3 \%$ in the placebo group (95\% CI 33.7-61.2), 28.8\% in the $5 \mathrm{mg} / \mathrm{h}$ clazosentan group (95\% CI 17.1-43.1), and $14.3 \%$ in the $10 \mathrm{mg} / \mathrm{h}$ clazosentan group (95\% CI 5.9-27.2; Fig. 6). Statistical testing indicated significantly lower occurrence of $\mathrm{M} / \mathrm{M}$ events in the $10 \mathrm{mg} / \mathrm{h}$ clazosentan group compared with placebo $(p=0.0003)$. M/M events are summarized in Figure 6. The occurrences of vasospasm-related cerebral infarction and DIND, as well as the need for rescue therapy were significantly lower in the $10 \mathrm{mg} / \mathrm{h}$ clazosentan group compared with placebo (Fig. 6). 
Table 1. Patient demographics and disease characteristics

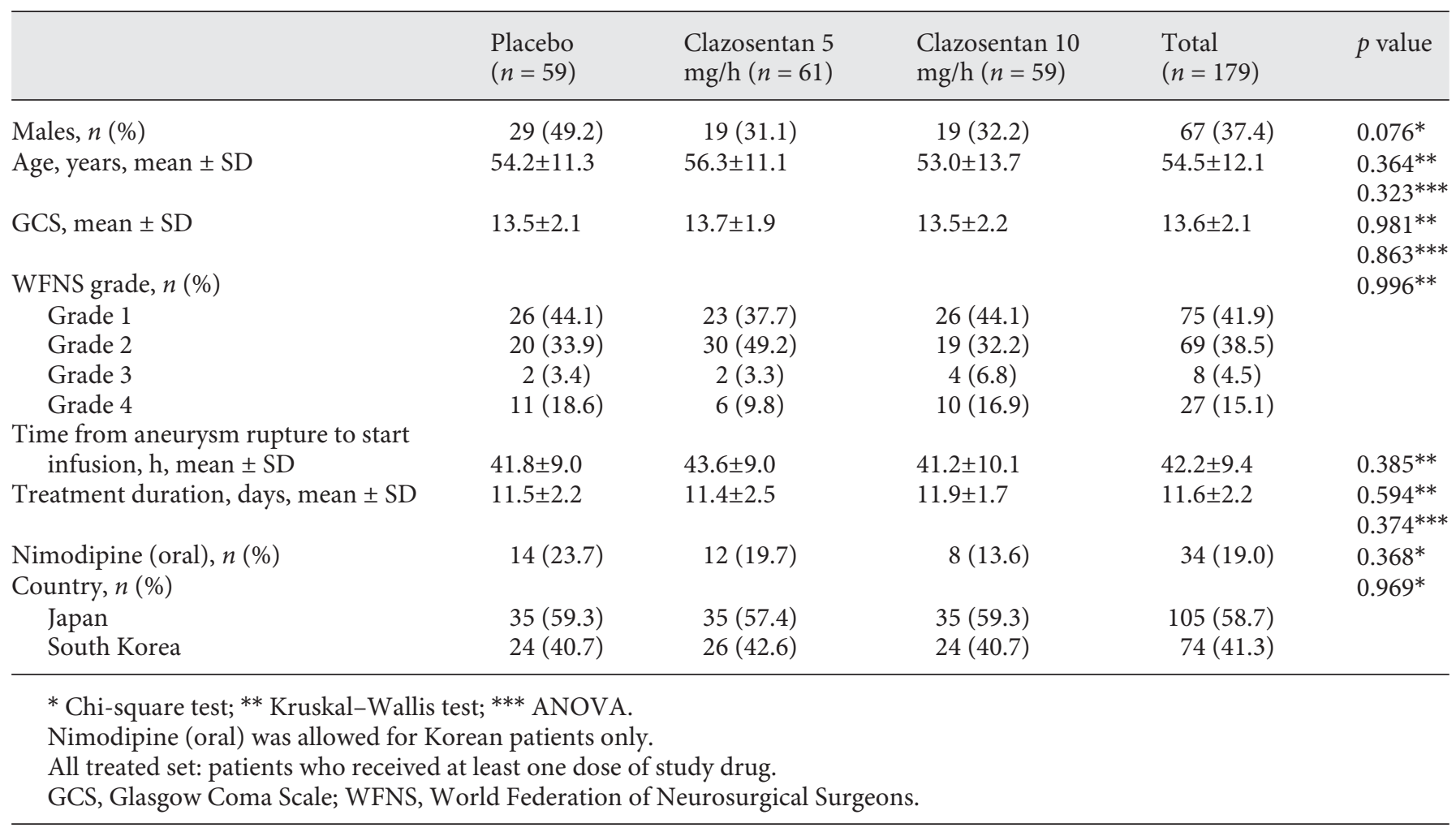

\section{Pharmacokinetics}

Clazosentan pharmacokinetics was assessed in $111 \mathrm{pa}-$ tients. The plasma clazosentan concentration in the 5 and $10 \mathrm{mg} / \mathrm{h}$ dose groups were 163.4 and $331.4 \mathrm{ng} / \mathrm{mL}$, respectively. There was no difference in plasma concentration between Japanese and Korean patients $(p=0.2830)$.

\section{Safety}

Drug administration was discontinued due to adverse events in $8.3,10.0$, and $6.8 \%$ of the patients in the placebo, $5 \mathrm{mg} / \mathrm{h}$ clazosentan, and $10 \mathrm{mg} / \mathrm{h}$ clazosentan groups, respectively. No differences in the total numbers of adverse events were observed between the 3 treatment groups.

Adverse events that were reported in 10 or more patients in all groups were cerebral vasospasm (56.7, 33.3, and $18.6 \%$ in the placebo, $5 \mathrm{mg} / \mathrm{h}$ clazosentan, and $10 \mathrm{mg} / \mathrm{h}$ clazosentan groups, respectively) and headache $(23.3,21.7$, and $25.4 \%$ in the placebo, $5 \mathrm{mg} / \mathrm{h}$ clazosentan, and $10 \mathrm{mg} / \mathrm{h}$ clazosentan groups, respectively). Lung complications were recorded in $11.7,25.0$, and $20.3 \%$ of patients in the placebo, $5 \mathrm{mg} / \mathrm{h}$ clazosentan, and $10 \mathrm{mg} / \mathrm{h}$ clazosentan groups, respectively. Hypotension was observed in $0,5.0$, and $5.1 \%$, respectively, and anemia was observed in 10.0, 18.3, and $13.6 \%$, respectively.

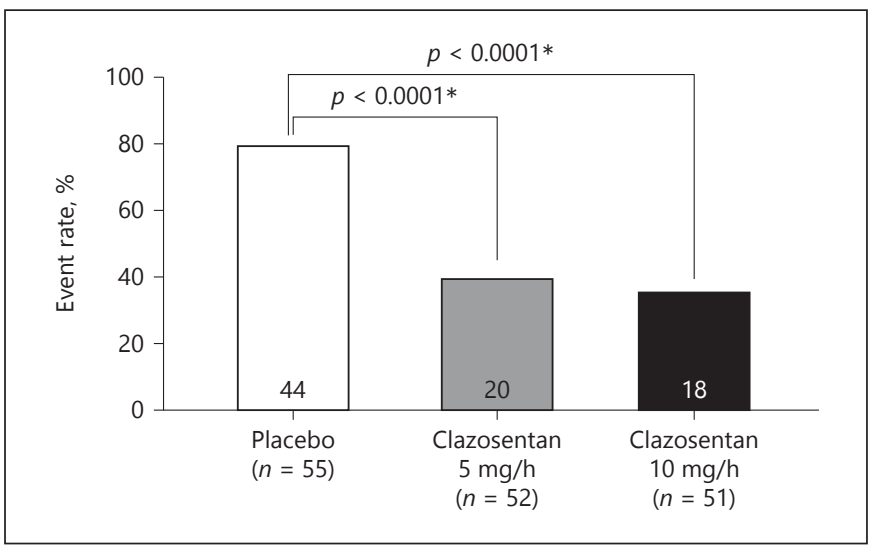

Fig. 4. Moderate-to-severe cerebral vasospasm (per-protocol set). Statistical multiplicity was adjusted using the Bonferroni-Holm method. Fisher's exact test ( $\alpha=0.025)$ was performed to assess the occurrence of cerebral vasospasm.

The percentages of patients who had died by 12 weeks after aSAH onset were $1.7 \%$ in the placebo group (1 patient due to carotid artery occlusion and vasospasm), $1.7 \%$ in the $5 \mathrm{mg} / \mathrm{h}$ clazosentan group (1 patient due to cerebral infarction), and $3.4 \%$ (2 patients due to cerebral infarction) in the $10 \mathrm{mg} / \mathrm{h}$ clazosentan group.
Cerebrovasc Dis 2017;44:59-67
DOI: $10.1159 / 000475824$
Fujimura/Joo/Kim/Hatta/Yokoyama/ Tominaga 


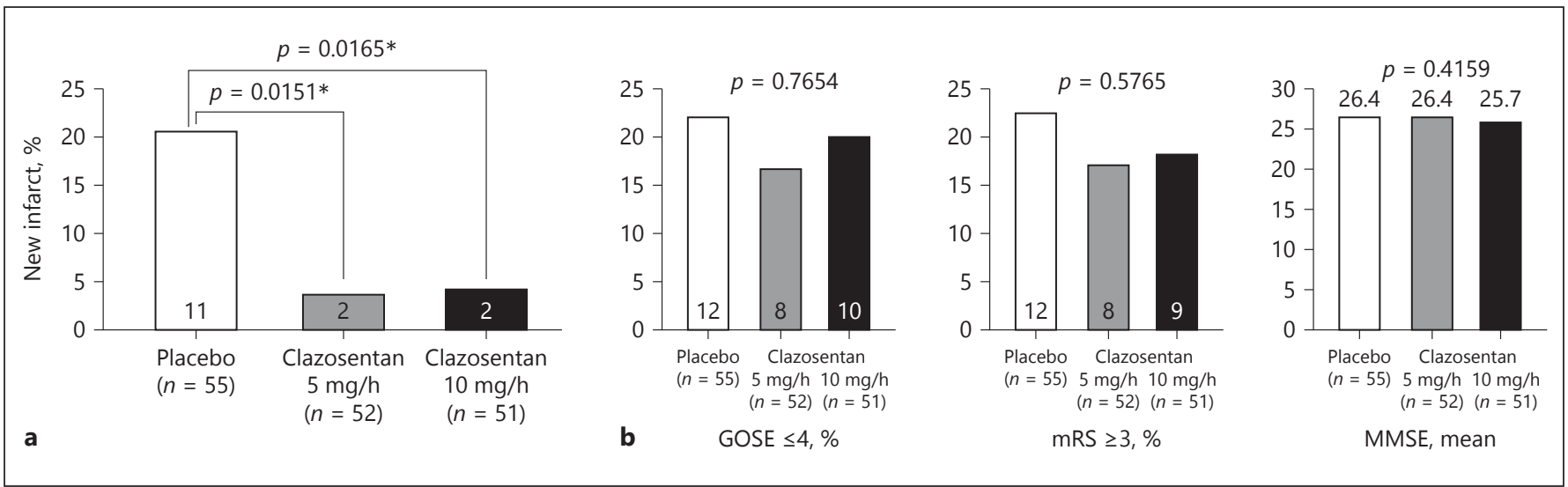

Fig. 5. Secondary endpoints (a) incidence of vasospasm-related cerebral infarction (PPS), estimated using Fisher's exact test; (b) incidence of poor outcome (GOSE $\leq 4, \mathrm{mRS} \geq 3$ ), estimated using the Cochran-Armitage trend test. Cognitive function (MMSE) at week 12 (PPS), estimated using the Jonckheere-Terpstra test. PPS, per-protocol set; GOSE, Glasgow Outcome Scale-Extension; mRS, modified Rankin Scale; MMSE, Mini-Mental State Examination.

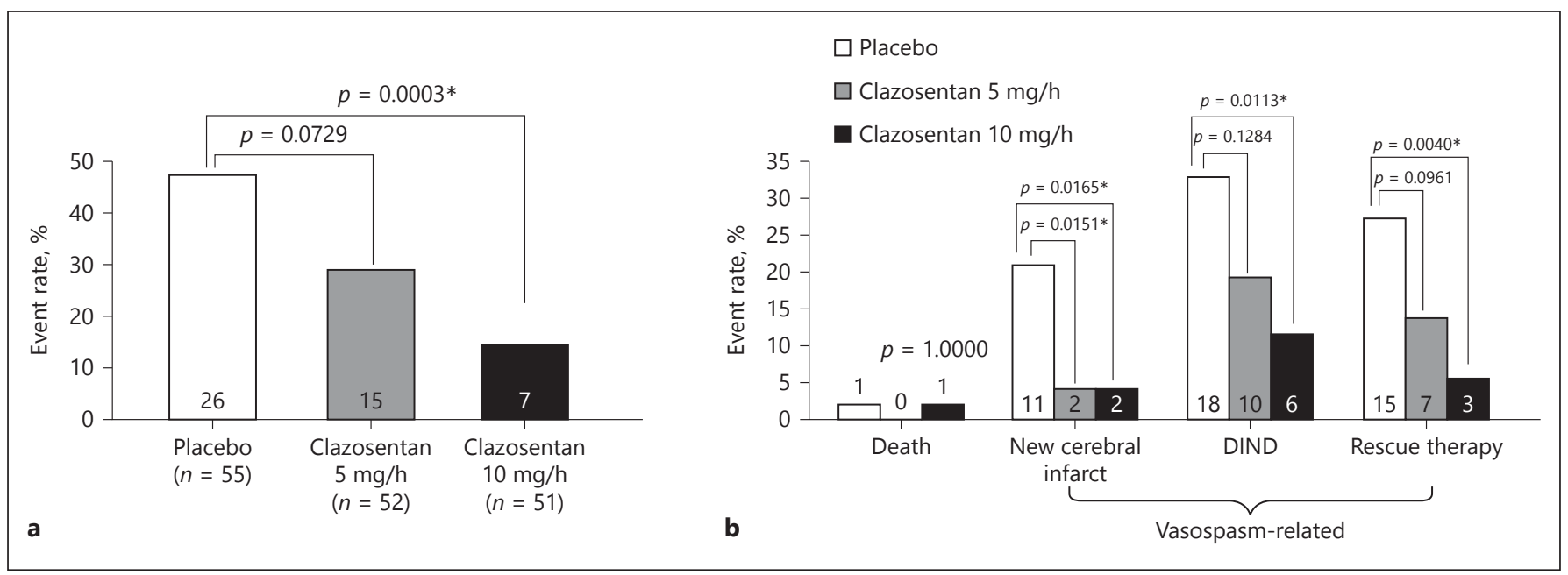

Fig. 6. Exploratory endpoints (a) overall incidence of death and vasospasm-related morbidity (PPS), estimated using Fisher's exact test; (b) incidence of death and each component of vaso-

\section{Discussion}

This study demonstrated, for the first time, that clazosentan prevents cerebral vasospasm and subsequent cerebral infarction after clipping surgery for aSAH in Japanese and Korean patients. There were no statistically significant differences between the placebo and clazosentan groups in GOSE and mRS scores 12 weeks after aSAH. These latter findings are in agreement with those previously reported in the CONSCIOUS-1, 2, and 3 trials with clazosentan [7-9]. It was observed in this study that among the secondary and exploratory endpoints, the in-

Clazosentan Prevents Cerebral Vasospasm after Clipping Surgery for aSAH spasm-related morbidity (PPS), estimated using Fisher's exact test. PPS, per-protocol set; DIND, delayed ischemic neurological deficit.

cidence of cerebral infarction associated with vasospasm and the M/M events within 6 weeks after aSAH were significantly reduced in the $10 \mathrm{mg} / \mathrm{h}$ clazosentan group compared to the placebo group. This result was distinct from that of CONSCIOUS-2 where no significant difference in the incidence of $\mathrm{M} / \mathrm{M}$ was observed between the placebo and the $5 \mathrm{mg} / \mathrm{h}$ clazosentan groups [9]. Thus, it is conceivable that the higher dose of clazosentan used in this study contributed, at least in part, to the favorable effect on cerebral infarction and $\mathrm{M} / \mathrm{M}$ events after aSAH.

The lack of statistically significant improvements in outcomes at 12 weeks despite the preventive effect of 
clazosentan on vasospasm could be related to a number of factors. First, the number of patients included in this study was not sufficient to conduct a prognostic evaluation 12 weeks after aSAH onset. For instance, it has been reported that a sample size of over 5,000 patients is necessary to fully evaluate drug efficacy based on mRS scores obtained up to 3 months after SAH onset [15]. Second, the rate at which rescue therapy was employed in the placebo group was approximately twice as high as that in the $5 \mathrm{mg} / \mathrm{h}$ clazosentan group, and almost 4 times higher than that in the $10 \mathrm{mg} / \mathrm{h}$ clazosentan group. Therefore, it is conceivable that the higher use of rescue therapy could have potentially compensated the deleterious effect of vasospasm especially in the placebo group, thereby diluting the treatment effect on the outcome at 12 weeks.

This study was conducted exclusively in large-volume centers, where strong rescue therapies for vasospasm such as interventional angioplasty and/or intra-arterial vasodilator infusion are available almost all the time, but these are not treatments that can be easily or rapidly administered at all medical facilities. Furthermore, intraarterial administration of a vasodilator could result in systemic hypotension, especially when administered at high doses $[16,17]$, which are associated with a risk of deterioration of the ischemic condition by vasospasm. Taken together with the high medical costs of rescue therapies [18], we believe that clazosentan could significantly contribute to the management of vasospasm in patients with aSAH, not only in large-volume centers but also in general clinical practice.

It is noteworthy that the incidence of cerebral vasospasm in this study was similar in both the 5 and $10 \mathrm{mg} / \mathrm{h}$ clazosentan treatment groups, while the incidence of DIND associated with vasospasm was significantly reduced in clazosentan-treated patients in a dose-dependent manner. It has been reported previously that microcirculatory vasospasm and endothelial cell injury associated with platelet adhesion could participate in the development of DIND [19]. It is therefore conceivable that treatment with $10 \mathrm{mg} / \mathrm{h}$ clazosentan in the current study further reduced the incidence of DIND by preventing the small-vessel vasospasm and/or blocking endothelial cell damage. However, it has been reported that clazosentan might expand major arteries but not small peripheral vessels [8]. A previous study has also suggested that an endothelin receptor antagonism could prevent vascular damage, endothelial dysfunction, and cerebral edema, and may lead to organ protection in hypertensive rats [20]. Further investigation of cerebral hemodynam- ics using positron emission tomography or single photon emission computed tomography could address this important issue.

This study has 2 key limitations. First, only aSAH patients who underwent clipping surgery were included, so it cannot robustly be determined whether these results can be reproduced in patients undergoing endovascular coiling. Suzuki and Taki [21] reported no difference in the occurrence of symptomatic cerebral vasospasm after clipping and coiling procedures in patients with aSAH, and a previous 3-year follow-up study has shown no difference in prognosis after either procedure [22]. The effect of clazosentan on symptomatic cerebral vasospasm after endovascular coiling therefore requires verification in future studies. Second, we do not rule out the possibility that the interpretation of the current study data could be affected in part by the fact that the primary efficacy analysis was based on the PPS. Nevertheless, findings from the ATS analysis confirmed the robustness of the primary analysis.

\section{Conclusions}

This clinical trial conducted in Japanese and Korean patients indicates that treatment with clazosentan at doses of 5 or $10 \mathrm{mg} / \mathrm{h}$ can markedly decrease the occurrence of cerebral vasospasm following aSAH, and may improve outcomes after the onset of aSAH by preventing cerebral infarction.

\section{Acknowledgments}

We would like to express our appreciation to all those who contributed to the clinical trial, including the IRC, the Data Safety Monitoring Board, the principal investigators, the sub-investigators, and the clinical research coordinators. The IRC members were Dr. Makoto Sasaki, Dr. Izumi Nagata, Dr. Takashi Watanabe, Dr. Kiyohiro Houkin, and Dr. Hiroyuki Kinouchi. The Data Safety Monitoring Board members were Dr. Tomio Sasaki, Dr. Yutaka Akae, and Dr. Nobuhito Saito. The principal investigators at sites where the trial was conducted were: Chonnam National University Hospital (Dr. Tae-Sun Kim), Gangnam Severance Hospital (Dr. Jin-Yang Joo), Saiseikai Kumamoto Hospital (Dr. Toru Nishi), Yeongnam University Medical Center (Dr. Chul-Hoon Chang), Baba Memorial Hospital (Dr. Hidefuku Gi), Kohnan Hospital (Dr. Takashi Inoue), Yamaguchi University Hospital (Dr. Michiyasu Suzuki), Yamagata City Hospital Saiseikan (Dr. Sinjiro Saito), Samsung Medical Center (Dr. Jong-Soo Kim), Sendai Medical Center (Dr. Hiroshi Uenohara), Dokkyo Medical University Hospital (Dr. Phyo Kim), Nakamura Memorial Hospital (Dr. Jyoji Na-
66

Cerebrovasc Dis 2017;44:59-67

DOI: $10.1159 / 000475824$
Fujimura/Joo/Kim/Hatta/Yokoyama/

Tominaga 
kagawara), Inha University Hospital (Dr. Hyeon-Seon Park), Hirosaki University Hospital (Dr. Kenichiro Asano), Aomori Prefectural Central Hospital (Dr. Tatsuya Sasaki), Showa General Hospital (Dr. Kazuo Tsutsumi), Yonsei University, Wonju Severance Christian Hospital (Dr. Kum Whang), Nagasaki Medical Center (Dr. Hiroshi Baba), Fujita Health University Hospital (Dr. Yoko Kato), Iwate Prefectural Central Hospital (Dr. Takayuki Sugawara), Shin Koga Hospital (Dr. Tsutomu Hitotsumatsu), Soon Chun Hyang University Hospital Bucheon (Dr. Bum-Tae Kim), The Catholic University of Korea, Seoul St. Mary's Hospital (Dr. Kwan-Sung Lee), Saitama Red Cross Hospital (Dr. Uichi Kaneko), Okayama Red Cross General Hospital (Dr. Keisuke Onoda), Ajou University Hospital (Dr. Yong Cheol Lim), Osaka University Hospital (Dr. Toshiyuki Fujinaka), Tottori University Hospital (Dr. Takashi Watanabe), Aso Iizuka Hospital (Dr. Yoshihiro Natori), and Iwate Medical University Hospital (Dr. Kuniaki Ogasawara). The statistical analyses and medical writing were supported by Actelion Pharmaceuticals Japan Ltd. The clazosentan blood con- centrations were measured by Inovalab AG (Switzerland). Matthew Reilly PhD at InTouch Medical Ltd. provided editorial support in the preparation of this manuscript, funded by Actelion.

\section{Source of Funding}

Actelion Pharmaceuticals Japan Ltd. provided the funding for this clinical trial. The sponsor was involved in the trial design, data collection, and data analysis and interpretation.

\section{Disclosure Statement}

M.F. and T.T. have received consultancy fees from Actelion Pharmaceuticals Japan Ltd. M.H. and Y.Y. are employees of Actelion Pharmaceuticals Japan Ltd.

\section{References}

1 de Oliveira JG, Beck J, Ulrich C, Rathert J, Raabe A, Seifert V: Comparison between clipping and coiling on the incidence of cerebral vasospasm after aneurysmal subarachnoid hemorrhage: a systematic review and metaanalysis. Neurosurg Rev 2007;30:22-30; discussion 30-31.

2 Connolly ES Jr, Rabinstein AA, Carhuapoma JR, Derdeyn CP, Dion J, Higashida RT, Hoh BL, Kirkness CJ, Naidech AM, Ogilvy CS, Patel AB, Thompson BG, Vespa P; American Heart Association Stroke Council; Council on Cardiovascular Radiology and Intervention; Council on Cardiovascular Nursing; Council on Cardiovascular Surgery and Anesthesia; Council on Clinical Cardiology: Guidelines for the management of aneurysmal subarachnoid hemorrhage: a guideline for healthcare professionals from the American Heart Association/American Stroke Association. Stroke 2012;43:1711-1737.

3 The Japan Stroke Society: Japanese Guidelines for the Management of Stroke 2015. Tokyo, Kyowakikaku, 2015 (in Japanese).

4 Cosentino F, Katusic ZS: Does endothelin-1 play a role in the pathogenesis of cerebral vasospasm? Stroke 1994;25:904-908.

5 Tippler B, Herbst C, Simmet T: Evidence for the formation of endothelin by lysed red blood cells from endogenous precursor. Eur J Pharmacol 1994;271:131-139.

6 Yanagisawa M, Kurihara H, Kimura S, Tomobe Y, Kobayashi M, Mitsui Y, Yazaki Y, Goto K, Masaki T: A novel potent vasoconstrictor peptide produced by vascular endothelial cells. Nature 1988;332:411-415.

7 Macdonald RL, Kassell NF, Mayer S, Ruefenacht D, Schmiedek P, Weidauer S, Frey A, Roux S, Pasqualin A; CONSCIOUS-1 Investigators: Clazosentan to overcome neurological ischemia and infarction occurring after subarachnoid hemorrhage
(CONSCIOUS-1): randomized, double-blind, placebo-controlled phase 2 dose-finding trial. Stroke 2008;39:3015-3021.

8 Macdonald RL, Higashida RT, Keller E, Mayer SA, Molyneux A, Raabe A, Vajkoczy P, Wanke I, Bach D, Frey A, Nowbakht P, Roux S, Kassell N: Randomized trial of clazosentan in patients with aneurysmal subarachnoid hemorrhage undergoing endovascular coiling. Stroke 2012;43:1463-1469.

9 Macdonald RL, Higashida RT, Keller E, Mayer SA, Molyneux A, Raabe A, Vajkoczy P, Wanke I, Bach D, Frey A, Marr A, Roux S, Kassell N: Randomised trial of clazosentan, an endothelin receptor antagonist, in patients with aneurysmal subarachnoid hemorrhage undergoing surgical clipping (CONSCIOUS-2). Acta Neurochir Suppl 2013;115:27-31.

10 Walpole SC, Prieto-Merino D, Edwards P, Cleland J, Stevens G, Roberts I: The weight of nations: an estimation of adult human biomass. BMC Public Health 2012;12:439.

11 Graham DI, Macpherson P, Pitts LH: Correlation between angiographic vasospasm, hematoma, and ischemic brain damage following SAH. J Neurosurg 1983;59:223-230.

12 Weidauer S, Lanfermann H, Raabe A, Zanella F, Seifert V, Beck J: Impairment of cerebral perfusion and infarct patterns attributable to vasospasm after aneurysmal subarachnoid hemorrhage: a prospective MRI and DSA study. Stroke 2007;38:1831-1836.

13 van Giersbergen PL, Gunawardena KA, Dingemanse J: Influence of ethnic origin and sex on the pharmacokinetics of clazosentan. J Clin Pharmacol 2007;47:1374-1380.

14 Vajkoczy P, Meyer B, Weidauer S, Raabe A, Thome C, Ringel F, Breu V, Schmiedek P: Clazosentan (AXV-034343), a selective endothelin A receptor antagonist, in the prevention of cerebral vasospasm following severe aneurysmal subarachnoid hemorrhage: re- sults of a randomized, double-blind, placebocontrolled, multicenter phase IIa study. I Neurosurg 2005;103:9-17.

15 Kreiter KT, Mayer SA, Howard G, Knappertz V, Ilodigwe D, Sloan MA, Macdonald RL: Sample size estimates for clinical trials of vasospasm in subarachnoid hemorrhage. Stroke 2009; 40:2362-2367.

16 Enomoto Y, Yoshimura S, Yamada K, Iwama $\mathrm{T}$ : Convulsion during intra-arterial infusion of fasudil hydrochloride for the treatment of cerebral vasospasm following subarachnoid hemorrhage. Neurol Med Chir (Tokyo) 2010; 50:7-11; discussion 11-12.

17 Tachibana E, Harada T, Shibuya M, Saito K, Takayasu M, Suzuki Y, Yoshida J: Intra-arterial infusion of fasudil hydrochloride for treating vasospasm following subarachnoid haemorrhage. Acta Neurochir (Wien) 1999;141:13-19.

18 Macdonald RL: Delayed neurological deterioration after subarachnoid haemorrhage. Nat Rev Neurol 2014;10:44-58.

19 Suzuki H, Kawakita F, Liu L, Ichikawa N, Fujimoto M, Shiba M: Current concepts of delayed cerebral ischemia after aneurysmal subarachnoid hemorrhage. Jpn J Neurosurg 2015; 24:232-238.

20 Callera G, Tostes R, Savoia C, Muscara MN, Touyz RM: Vasoactive peptides in cardiovascular (patho)physiology. Expert Rev Cardiovasc Ther 2007;5:531-552.

21 Suzuki H, Taki W; Prospective Registry of Subarachnoid Aneurysms Treatment (PRESAT) Group: Effect of aneurysm treatment modalities on cerebral vasospasm after aneurysmal subarachnoid hemorrhage. Acta Neurochir Suppl 2013;115:99-105.

22 Spetzler RF, McDougall CG, Albuquerque FC, Zabramski JM, Hills NK, Partovi S, Nakaji $\mathrm{P}$, Wallace RC: The barrow ruptured aneurysm trial: 3-year results. J Neurosurg 2013; 119:146-157. 